\title{
DETERMINATION OF OPTIMAL ELASTIC SPRINGS FOR CANTILEVER BEAMS SUPPORTED BY ELASTIC FOUNDATION
}

\author{
E. Aydin", B. Öztürk ${ }^{* * *}$, M. Dutkiewicz ${ }^{* * *}$
}

\begin{abstract}
In this study, the optimum distribution of the elastic springs in which a built-in cantilever beam is seated, so as to minimize the shear force on the support of the beam, is investigated. The Fourier transform is applied to the vibration equation of the beam written in the time domain and is shown by the structural behaviour transfer function which is made independent from the external influence. For the first and second modes of beam, the optimum locations and amounts of the springs were investigated so that the shear force transfer function amplitude was minimal. Stiffness coefficients of springs are taken as design variables. There are active constraints on the sum of the spring coefficients taken as design variables and passive constraints on each of them as the upper and lower bounds. Optimality criteria are derived using the Lagrange Multipliers method. The gradient information required for solving the optimization problem is analytically derived. Numerical results show that the aimed method is quite effective in finding optimum spring stiffness coefficients.
\end{abstract}

Keywords: optimal springs, shear force minimization, beam vibration, optimal stiffness, transfer functions

\section{Introduction}

Beams are the basic structural elements used in many different engineering applications. Many researchers have invastigated problems with beams subjected to static and dynamic loads. Some basic studies on free vibrations of beams can be given as follows. Timoshenko et al. (1974) showed free vibration frequencies of the beams based on the floor of the Winkler composed of elastic springs. Chung et al. (1993) have shown an analytical solution method to find the natural frequencies of beams that are constrained from the middle, elastically supported on both sides. Aristizabal (1993) studied the free vibration of prismatic beams. Lee et al. (1999) studied the free vibrations of piles partially buried in the elastic layer. In Lee et al. (2002), a new method has been developed to find the buckling loads and natural frequencies of prismatic columns supported by an elastic spring in the centre, with the aim of finding free vibrations for general boundary conditions of beams.

Liu et al. (1996) derived closed-form frequency sensitivities based on the Rayleigh principle using the Lagrange Multipliers method. Sinha and Friswell (2001) have applied a support location to element shape functions to form a global stiffness matrix. Won and Park (1998) showed optimal support placement due to the stiffness of the support. A optimization method has been shown by Takewaki (1998) to use transfer functions to optimize the location and amount of dampers in an embedded beam sitting on viscous dampers.

In this study, the optimization of the locations and amounts of the springs was investigated to minimize the support shear force corresponding to the first and second modes of a cantilever beam supported by

\footnotetext{
*Assoc. Prof .Ersin Aydin, Faculty of Engineering, Department of Civil Engineering,Nigde Ömer Halisdemir University, Nigde, Turkey, eaydin@ohu.edu.tr

** Assoc. Prof. Baki Öztürk,Faculty of Engineering, Department of Civil Engineering, Hacettepe University, Ankara Turkey, bakiozturk@hacettepe.edu.tr

*** Dr. Eng. Maciej Dutkiewicz, Faculty of Civil, Architecture and Environmental Engineering, Department of Building Construction, University of Science and Technology in Bydgoszcz, Al. Prof. S. Kaliskiego 7, 85-796 Bydgoszcz PL, e-mail: macdut@utp.edu.pl
} 
elastic springs. The Fourier transform is applied to the motion equation written in the time domain and is expressed by transfer functions. SDSA (Stepest Direction Search Algorithm) method which is used by Takewaki (1998) to solve the optimum spring problem is used.

\section{Theoretical background of the analysed problem}

In the case where the cantilever beam subjected to a bearing action shown in Fig. 1 does not have springs, the motion equation can be written as follows:

$$
\boldsymbol{M} \ddot{\boldsymbol{u}}(t)+\boldsymbol{C} \dot{\boldsymbol{u}}(t)+\boldsymbol{K u}(t)=-\boldsymbol{M r} \ddot{u}_{g}(t)
$$

Where $\boldsymbol{M}, \boldsymbol{C}$ and $\boldsymbol{K}$ present mass, structural damping and stiffness matrices, respectively $\ddot{\boldsymbol{u}}(t)$, $\dot{\boldsymbol{u}}(t)$ and $\boldsymbol{u}(t)$ are acceleration, velocity and displacement vectors, respectively. The $\boldsymbol{r}$ denotes influence vector, $\ddot{u}_{g}(t)$ is defined as base acceleration.

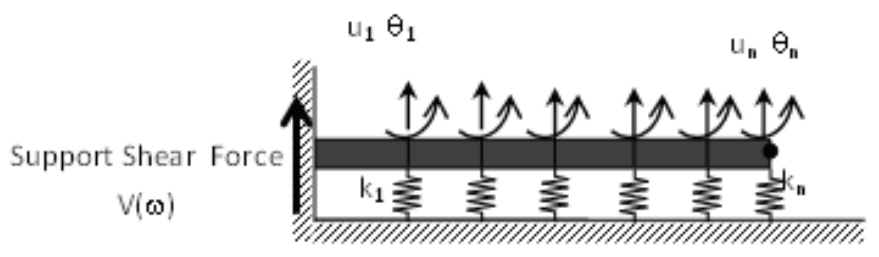

Fig.1. Cantilever beam based on elastic springs

If the Fourier transformation is applied to Eq. (1), $\boldsymbol{U}(\omega)$ and $\ddot{U}_{g}(\omega)$ is the Fourier Transformation of $\boldsymbol{u}(t)$ and $\ddot{u}_{g}(t)$. Eq $(1)$ is rewritten as

$$
\left(\boldsymbol{K}+i \omega \boldsymbol{C}-\omega^{2} \boldsymbol{M}\right) \boldsymbol{U}(\omega)=-\boldsymbol{M r} \ddot{U}_{g}(\omega)
$$

where $\omega$ denotes the circular frequency of the effect, and $i$ denotes $\sqrt{-1}$. As shown in Fig. 1, if the beam is supported by elastic springs, Eq. (2) is changed to

$$
\left(\left(\boldsymbol{K}+\boldsymbol{K}_{\boldsymbol{a d}}\right)+i \omega \boldsymbol{C}-\omega^{2} \boldsymbol{M}\right) \boldsymbol{U}_{\boldsymbol{a d}}(\omega)=-\boldsymbol{M r} \ddot{U}_{g}(\omega)
$$

Here $K_{a d}$ shows the stiffness matrix of the inserted springs. $\boldsymbol{U}_{a d}(\omega)$ is the Fourier Transform of the displacements after the springs are added. A new parameter (Takewaki 1998) given as follow,

$$
\widehat{\boldsymbol{U}}(\omega)=\frac{\boldsymbol{U}_{a d}(\omega)}{\ddot{U}_{g}(\omega)}
$$

If $\omega=\omega_{n}$ is taken here, the ground motion will be defined as a harmonic motion with frequency $\omega_{n}$. Using Eq. (4), Eq. (3) can be rewritten as

$$
\boldsymbol{A} \widehat{\boldsymbol{U}}\left(\omega_{n}\right)=-\boldsymbol{M r}
$$

Make $\widehat{\boldsymbol{U}}\left(\omega_{n}\right)$ here represents the transfer function of the displacements calculated at the $\mathrm{n}^{\text {th }}$ natural frequency. The matrix $\boldsymbol{A}$, which contains the design variable spring stiffness coefficients $\left(\mathrm{k}_{1}, \mathrm{k}_{2}, \ldots, \mathrm{k}_{\mathrm{n}}\right.$, ). Matrix $\boldsymbol{A}$ is given as

$$
\boldsymbol{A}=\left(\boldsymbol{K}+\boldsymbol{K}_{\boldsymbol{a d}}\right)+i \omega_{n} \boldsymbol{C}-\omega_{n}^{2} \boldsymbol{M}
$$

Here $\boldsymbol{K}, \boldsymbol{M}$ and $\boldsymbol{C}$ are known. The $\boldsymbol{K}_{\boldsymbol{a d}}$ matrix containing the design variables will be found. If Eq. (5) is rewritten as follows,

$$
\widehat{\boldsymbol{U}}\left(\omega_{n}\right)=-\boldsymbol{A}^{-1} \boldsymbol{M r}
$$

transfer function displacement vector is found. The force vector of the transfer function is obtained by multiplying the displacement vector by the stiffness matrix and the transfer function as follows.

$$
\boldsymbol{F}\left(\omega_{n}\right)=-\left(\boldsymbol{K}+\boldsymbol{K}_{\boldsymbol{a d}}\right) \boldsymbol{A}^{-1} \boldsymbol{M r}
$$

\section{Optimization Problem}

The mathematical representation of the objective function is used

Here, the objective function $f$ is defined as follows:

$$
\text { Min } f(\boldsymbol{k})=f\left(k_{1}, k_{2}, k_{3}, \ldots, k_{n}\right)
$$




$$
f(\boldsymbol{k})=\sum_{j=1}^{n}\left|F_{j}\left(\omega_{i}\right)\right|(\mathrm{i}=\text { mode number })
$$

Where $\left|F_{j}\left(\omega_{i}\right)\right|$ represents the absolute value of the transfer function amplitude of the $\mathrm{j}^{\text {th }}$ force against to $\mathrm{j}^{\text {th }}$ degree of freedom for $\mathrm{i}^{\text {th }}$ mode. Also for each spring coefficient, passive constraints given as follow

$$
0 \leq k_{i} \leq \bar{k}_{i} \quad(\mathrm{i}=1,2, \ldots, \mathrm{N})
$$

Where $\bar{k}_{i}$ is the upper limit of the $\mathrm{i}^{\text {th }}$ spring constant. In the sum of the spring coefficients an active constraint can be written as

$$
\sum_{i=1}^{N} k_{i}=\bar{K}
$$

Here $\bar{K}$ is the sum of the spring coefficients to be added.

\section{Optimality Criteria}

Optimality criteria can be derived using the Lagrange Multipliers method. Depending on the generalized Lagrangian functional objective function and the Lagrange multipliers $(\lambda, \mu$ and $v)$ is given as

$$
L\left(k_{i}, \lambda, \mu_{i}, v_{i}\right)=f(\boldsymbol{k})+\lambda\left(\sum_{i=1}^{n}\left(k_{i}-\bar{K}\right)\right)+\sum_{i=1}^{n} \mu_{i}\left(0-k_{i}\right)+\sum_{i=1}^{N} v_{i}\left(k_{i}-\bar{k}_{i}\right)
$$

If Eq. (13) is derived from design variables and Lagrange Multipliers, optimality criteria can be written as

$$
\begin{gathered}
\frac{\partial f}{\partial k_{i}}+\lambda=0(i=1,2, \ldots, n) \quad 0<k_{i}<\bar{k}_{i} \\
\sum_{i=1}^{N} k_{i}-\bar{K}=0
\end{gathered}
$$

Here the partial derivative of objective function the according to design variable $k_{i}$ expressesas $\frac{\partial f}{\partial k_{i}}$. Eq. (14) for lower and upper constraints is changed as follows.

$$
\begin{array}{ll}
\frac{\partial f}{\partial k_{i}}+\lambda \geq 0 & k_{i}=0 \\
\frac{\partial f}{\partial k_{i}}+\lambda \leq 0 & k_{i}=\bar{k}_{i}
\end{array}
$$

For the solution of the problem, the method shown by Aydin (2014) was used. In Aydin (2014) study, he adapted the SDSA method given by Takewaki (1998) to the optimization of the springs. The derivation of the sensitivity equations in the optimization problem and the algorithm can be found from these sources.

\section{Numerical Example}

The $6 \mathrm{~m}$ long cantilever beam shown in Fig. 1 is modelled as a Timoshenko beam by dividing it into $1 \mathrm{~m}$ finite elements and assuming a linear angular displacement at each node. The shear modulus $\mathrm{G}=7.9410^{10}$ $\mathrm{N} / \mathrm{m}^{2}$, the correction factor $\mathrm{\kappa}=5 / 6$, the cross-sectional area $\mathrm{A}=0.05 \mathrm{~m}^{2}$, the moment of inertia $\mathrm{I}=2.0810^{-4}$ $\mathrm{m}^{4}$, density of material $\rho=7.810^{3} \mathrm{~kg} / \mathrm{m}^{3}$, the modulus of elasticity $\mathrm{E}=2.0610^{11} \mathrm{~N} / \mathrm{m}^{2}$, the total amount of stiffness was chosen as $\bar{K}=2282400 \mathrm{~N} / \mathrm{m}$ and $\Delta K=\bar{K} / 300$. A mass of $100 \mathrm{~kg}$ was also added to the end of the beam.

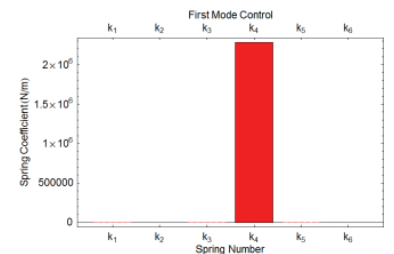

(a)

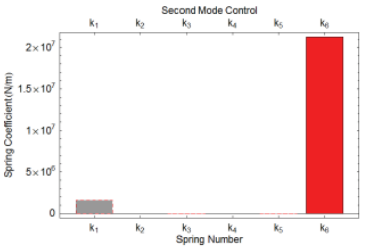

(b)

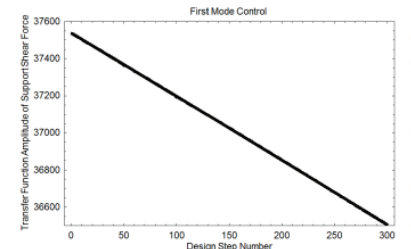

(c)

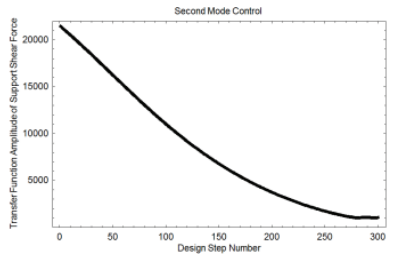

(d)

Fig.2. (a)-(b)Optimal spring placement for first and second mode (c)-(d) the variation of the transfer function of support shear force

Figures 2 (a) and (b) show the values and locations of optimum spring coefficients. To control the primary mode, it is necessary to place the total spring constant optimally at the fourth node. For the control of the second mode, placing a large part of the spring constant on the sixth node, while placing a first node on one part gives the optimum solution.Figures 2 (c) and (d) show us that the objective function falls for first and second modes according to the design step number. 


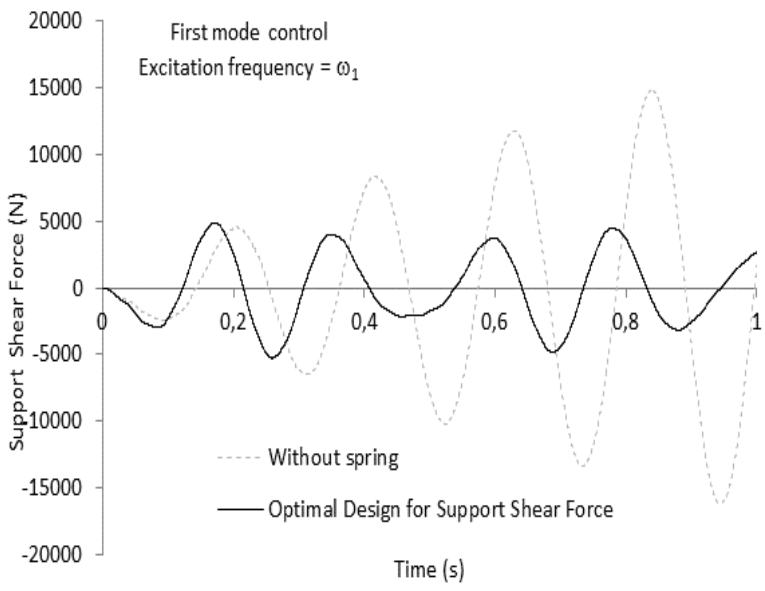

(a)

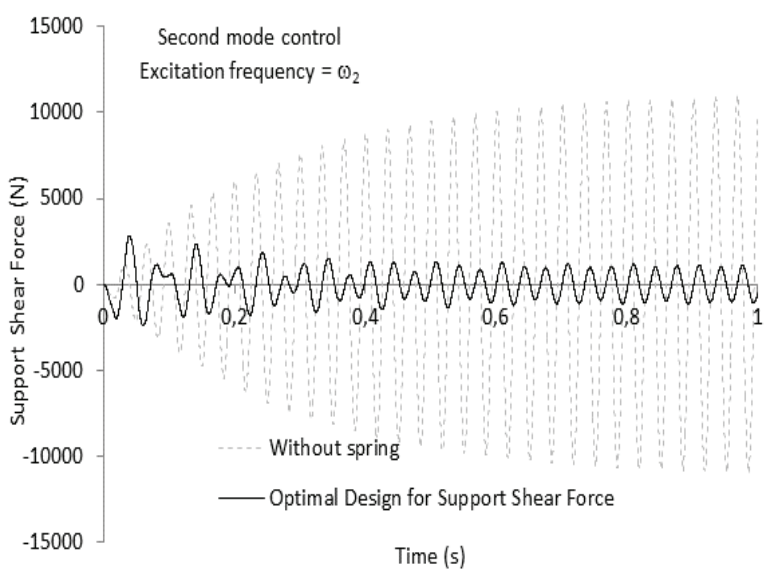

(b)

Fig.3. (a)-(b)Time histories of support shear force for first and second mode under resonance frequencies

As can be seen from Figs. 3 (a) and (b), the optimum design considerably reduce the shear force on the support for the first and second modes.

\section{Conclusion}

In the proposed study, optimum designs for the first two modes of cantilever beams based on elastic springs were investigated. Optimum designs have been found under the defined constraints, which are based on the shear force of the support. The results are shown in the time-domain calculations, which significantly reduce the shear force on the support. The vibrations of the cantilever beams were examined separately for the first mode and the second mode, and the different designs for both cases were placed in the middle. The work of giving considerable knowledge in engineering designs where supporting is important provides results that can be useful for designing beams based on an elastic foundation.

\section{References}

Aristizabal-Ochoa, J.D. (1993) Static, stability and vibration of non-prismatic beams and columns, Journal of Sound and Vibration, 62(3), 441455.

Aydın, E. (2014) Minimum Dynamic Response of Cantilever Beams Supported by Optimal Elastic Springs, Structural Engineering and Mechanics, 51(3), 377-402.

Chung, J.H., Joo, W.H. and Kim.K.C. (1993) Vibration and dynamic sensitivity analysis of a Timoshenko beam-column with elastically restrained ends and intermediate constraints, Journal of Sound and Vibration, 167(2), 209-215.

Lee, B.K., Jeong, J.S., Li, G.F. and Jin, T.K. (1999) Free vibrations of tapered piles embedded partially in an elastic foundation, Chinese Journal of Geotechnical Engineering, 21(5), 609- 613.

Lee, B.K., Lee. J.K., Lee, T.E. and Kim, S.G. (2002) Free vibrations of tapered beams with general boundary conditions, KSCE Journal of Civil Engineering, 6(3), 283-288.

Liu Z.S., Hu H.C. and Wang D.J. (1996) New method for deriving eigenvalue rate with respect to support location, AIAA J, 34, 864-866.

Sinha, J.K. and Friswell, M.I. (2001) The location of spring supports from measured vibration data, Journal of Sound and Vibration, 244, 137-153.

Takewaki, I. (1998) Optimal damper positioning in beams for minimum dynamic compliance, Comput Methods in Appl Mech and Engng, 156, 363-73.

Timoshenko, S.I, Young, D.H. and Weaver, W. (1974) Vibration Problems in Engineering, John Wiley \& Sons.

Won, K.M. and Park, Y.S. (1998) Optimal support position for a structure to maximize its fundamental natural frequency, Journal of Sound and Vibration, 213, 801-812. 